\title{
Segment Orders
}

\section{Csaba Biró • William T. Trotter}

Received: 14 September 2008 / Revised: 9 June 2009 / Accepted: 9 June 2009 /

Published online: 24 June 2009

(C) Springer Science+Business Media, LLC 2009

\begin{abstract}
We study two kinds of segment orders, using definitions first proposed by Farhad Shahrokhi. Although the two kinds of segment orders appear to be quite different, we prove several results suggesting that the are very much the same. For example, we show that the following classes belong to both kinds of segment orders: (1) all posets having dimension at most 3; (2) interval orders; and for $n \geq 3$, the standard example $S_{n}$ of an $n$-dimensional poset, all 1-element and ( $\left.n-1\right)$-element subsets of $\{1,2, \ldots, n\}$, partially ordered by inclusion.

Moreover, we also show that, for each $d \geq 4$, almost all posets having dimension $d$ belong to neither kind of segment orders. Motivated by these observations, it is natural to ask whether the two kinds of segment orders are distinct. This problem is apparently very difficult, and we have not been able to resolve it completely. The principal thrust of this paper is the development of techniques and results concerning the properties that must hold, should the two kinds of segment orders prove to be the same. We also derive equivalent statements, one version of which is a stretchability question involving certain sets of pseudoline arrangements. We conclude by proving several facts about continuous universal functions that would transfer segment orders of the first kind into segments orders of the second kind.
\end{abstract}

Keywords Segment order

C. Biró (凶)

Department of Mathematics, University of South Carolina, Columbia, SC 29208, USA

e-mail: biro@math.sc.edu

W.T. Trotter

School of Mathematics, Georgia Institute of Technology, Atlanta, GA 30332-0160, USA

e-mail: trotter@math.gatech.edu 


\section{Introduction}

We consider the euclidean plane equipped with a standard $x, y$ coordinate system and say a line segment $s$ in the plane is regular if it satisfies the following two properties:

(1) One end point of the segment is on the $x$-axis; and

(2) The slope of the line segment is positive.

Associated with a regular line segment $s$ is a triple $\left(s_{1}, s_{2}, s_{3}\right)$ of real numbers with $s_{2}>s_{1}$ and $s_{3}>0$ so that the "left" endpoint of $s$ is at $\left(s_{1}, 0\right)$ and the "right" endpoint of $s$ is at $\left(s_{2}, s_{3}\right)$.

Farhad Shahrokhi [11] proposed definitions for two partial orders on a family of regular line segments. These orders will be denoted by $\mathfrak{P}_{1}$ and $\mathfrak{P}_{2}$, respectively. They resemble the more general segment orders introduced by Pach and Törő́csik in [10].

\subsection{Segment Orders of the First Kind}

Let $s=\left(s_{1}, s_{2}, s_{3}\right)$ and $t=\left(t_{1}, t_{2}, t_{3}\right)$ be regular line segments. We say that $s>t$ in $\mathfrak{P}_{1}$ if

(i) $s_{1}<t_{1}$;

(ii) $s_{2}>t_{2}$; and

(iii) If $x_{0}$ is a real number and the vertical line $x=x_{0}$ intersects $s$ and $t$ at $\left(x_{0}, y_{1}\right)$ and $\left(x_{0}, y_{2}\right)$, respectively, then $y_{1}>y_{2}$.

More generally, we say that a poset $P$ is a segment order of the first kind when there is a function $S$ assigning to each element $u \in P$ a regular line segment $S(u)$ so that $u>v$ in $P$ if and only if $S(u)>S(v)$ in $\mathfrak{P}_{1}$. It is natural to abbreviate this statement just by saying that $P$ is a $\mathfrak{P}_{1}$ order. Also, we call the function $S$ a $\mathfrak{P}_{1}$ representation of $P$ and often do not distinguish between a $\mathfrak{P}_{1}$ order $P$ and a $\mathfrak{P}_{1}$ representation of $P$.

A poset $P$ is called an interval containment order when there exists a function $I$ assigning to each element $u$ of $P$ a non-degenerate closed interval $\left[a_{u}, b_{u}\right]$ of the real line so that $u \leq v$ in $P$ if and only if $\left[a_{u}, b_{u}\right] \subseteq\left[a_{v}, b_{v}\right]$. Segment orders of the first kind are a natural generalization of interval containment orders.

Proposition 1.1 Every interval containment order is a $\mathfrak{P}_{1}$ order.

Proof Let $I$ be a representation of an interval containment order $P$. Without loss of generality, ${ }^{1}$ we assume that all endpoints in the representation are distinct. Then the function $S$ defined by $S(u)=\left(a_{u}, b_{u}, b_{u}-a_{u}\right)$ is a representation of $P$ as a $\mathfrak{P}_{1}$ order.

\subsection{Segment Orders of the Second Kind}

Again let $s=\left(s_{1}, s_{2}, s_{3}\right)$ and $t=\left(t_{1}, t_{2}, t_{3}\right)$ be regular line segments. We say that $s>t$ in $\mathfrak{P}_{2}$ when conditions (i), (ii') and (iii) are satisfied, where conditions (i) and (iii) are

\footnotetext{
${ }^{1}$ We comment that we may assume that representations are in a "general position", whenever we are working with a finite poset. For example, intervals may be assumed to have distinct endpoints, segments have distinct heights and slopes, etc.
} 
just the same as before but now we have

(ii') $s_{2}<t_{2}$.

Analogously, we say that a poset $P$ is a segment order of the second kind (abbreviated to $P$ is a $\mathfrak{P}_{2}$ order) when there is a function $S$ assigning to each element $u \in P$ a regular line segment $S(u)$ so that $u>v$ in $P$ if and only if $S(u)>S(v)$ in $\mathfrak{P}_{2}$. Now, we will refer to the function $S$ as a $\mathfrak{P}_{2}$-representation of $P$.

\subsection{Interval Orders}

Recall that a poset $P$ is an interval order when there is a function $I$ associating with each element $u \in P$ a closed interval $I(u)=\left[a_{u}, b_{u}\right]$ on the real line so that $u<v$ in $P$ if and only $b_{u}<a_{v}$. Also, recall that the dual of a poset $P$, denoted $P^{d}$, is the poset on the same ground set with $u>v$ in $P^{d}$ if and only if $u<v$ in $P$. The dual of an interval order is also an interval order.

Proposition 1.2 Every interval order is a segment order of the second kind.

Proof Let $P$ be an interval order, and let $I$ be a function that assigns to each $u$ in $P$ an interval $I(u)=\left[a_{u}, b_{u}\right]$ of the real line so that $u>v$ in $P$ if and only if $b_{u}<a_{v}$. Without loss of generality, we may assume that the end points in the representation $I$ are distinct. Proceeding from left to right in the order of left endpoints, we may lift the interval $\left[a_{u}, b_{u}\right]$ to form a regular segment $\left[a_{u}, b_{u}, h_{u}\right]$ where the height $h_{u}$ is sufficiently large so that the resulting segment overlaps any segment associated with a point $v$ that is incomparable with $u$ in $P$ and has $a_{v}<a_{u}$.

\subsection{Central Segment Orders}

Shahroki's original definitions were not exactly the same as presented here. In particular, he considered only regular line segments $s=\left(s_{1}, s_{2}, s_{3}\right)$ for which $s_{1}<0$ and $s_{2}>0$. Accordingly, we say a regular segment $s=\left(s_{1}, s_{2}, s_{3}\right)$ is central if $s_{1}<0$ and $s_{2}>0$, and we let $\mathfrak{p}_{1}$ to denote the subclass of $\mathfrak{P}_{1}$ consisting of posets having representations using central segments. Posets in $\mathfrak{p}_{1}$ are called central segment orders of the first kind. Similarly, we can define $\mathfrak{p}_{2}$, the class of central segment orders of the second kind.

Of course, we have the trivial statements $\mathfrak{p}_{1} \subseteq \mathfrak{P}_{1}$ and $\mathfrak{p}_{2} \subseteq \mathfrak{P}_{2}$, but it is not at all clear if these containments are proper or not. In fact, the fundamental issue of attempting to distinguish between these four classes of segment orders will be the principal theme of this paper.

\subsection{Dimension and Shahroki’s Original Question}

Recall that the dimension of a poset $P$, denoted $\operatorname{dim}(P)$, is the least positive integer $d$ for which there exist $d$ linear orders $L_{1}, L_{2}, \ldots, L_{d}$ on the ground set of $P$ so that $u>v$ in $P$ if and only if $u>v$ in $L_{i}$ for each $i=1,2, \ldots, d$.

In the next section, we will prove the following two results, the first of which was known to Shahroki. 
Theorem 1.3 Every poset $P$ with $\operatorname{dim}(P) \leq 3$ is both a $\mathfrak{p}_{1}$ order and a $\mathfrak{p}_{2}$ order.

Theorem 1.4 For each $d \geq 4$, almost all posets $P$ with $\operatorname{dim}(P)=d$ are neither $\mathfrak{P}_{1}$ orders or $\mathfrak{P}_{2}$ orders, i.e., for each $d \geq 4$ and each $\varepsilon>0$, there exists an integer $n_{0} \geq 2 d$ so that if $n \geq n_{0}$ then among the posets with ground set $\{1,2, \ldots, n\}$ the ratio of posets that are either $\mathfrak{P}_{1}$ orders or $\mathfrak{P}_{2}$ orders divided by the total number of posets is less than $\varepsilon$.

So the following questions of Shahroki [11] are natural:

Question 1.5 Do there exist $\mathfrak{p}_{1}$ posets of arbitrarily large dimension? Do there exist $\mathfrak{p}_{2}$ posets of arbitrarily large dimension?

In the next section of the paper, we will answer Shahroki's question affirmatively by showing that there are posets of arbitrarily large dimension belonging to both $\mathfrak{p}_{1}$ and $\mathfrak{p}_{2}$.

\subsection{Interval Orders}

The reader should note that interval orders and interval containment orders are two very different classes of posets. In particular, interval containment posets are just the posets having dimension at most 2 , while interval orders can have arbitrarily large dimension. In fact, the dimension of the interval order $I_{n}$ determined by all $\left(\begin{array}{l}n \\ 2\end{array}\right)$ intervals with integer end points in $\{1,2, \ldots, n\}$ is known (see [6], for example) to satisfy the following asymptotic formula:

$$
\operatorname{dim}\left(P_{n}\right)=\lg \lg n+\left(\frac{1}{2}+o(1)\right) \lg \lg \lg n .
$$

The poset $I_{n}$ is called the canonical interval order.

When introducing the class $\mathfrak{P}_{2}$, we showed that all interval orders are $\mathfrak{P}_{2}$ orders, but the presented argument does not show that all interval orders are $\mathfrak{p}_{2}$ orders.

\section{Motivation and Background}

Theorems 1.3 and 1.4 seem to suggest that the four classes of segment orders may actually be the same. To provide further supporting evidence, we will also prove the following result.

Theorem 2.1 Every interval order is both a $\mathfrak{p}_{1}$ order and $a \mathfrak{p}_{2}$ order.

Definition 2.2 For each $n \geq 3$, the standard example $S_{n}$ is the poset whose ground set is $\left\{a_{1}, \ldots, a_{n}, b_{1}, \ldots, b_{n}\right\}$ with $b_{i}<a_{j}$ in $S_{n}$ if and only if $i \neq j$. The dimension of the standard example $S_{n}$ is $n$, and the removal of any point lowers the dimension to $n-1$. 
Note that the standard example $S_{n}$ is isomorphic to the family of all 1-element and $(n-1)$-element subsets of $\{1,2, \ldots, n\}$ partially ordered by inclusion.

Theorem 2.3 For each $n \geq 3$, the standard example $S_{n}$ belongs to both $\mathfrak{p}_{1}$ and $\mathfrak{p}_{2}$.

\subsection{The Removable Pair Conjecture}

One of the most fundamental results in dimension theory for posets is the following inequality of Hiraguchi [8]:

Theorem 2.4 For each $n \geq 4$, if $P$ is a poset with $n$ elements, then $\operatorname{dim}(P) \leq n / 2$.

Although several proofs of Hiraguchi's theorem have been found over the years, the following natural conjecture, posed by Trotter in [13], has remained open for more than 30 years.

Conjecture 2.5 (Removable Pair Conjecture) If $P$ is a poset on three or more points, then $P$ contains a pair of points whose removal decreases the dimension by at most 1 .

However, combining results from the literature on dimension theory, it is straightforward to verify the following theorem:

Theorem 2.6 Let $P$ be a poset on three or more points. Then $P$ contains a pair of points whose removal decreases the dimension by at most 1 if either of the following conditions hold:

(1) The dimension of $P$ is at most 3.

(2) $P$ is an interval order.

Accordingly, the four classes of segment orders are potentially very interesting classes to study in relation to the Removable Pair Conjecture.

\subsection{Distinct Class Conjecture}

Despite the evidence to the contrary presented in this paper, the authors firmly believe that the following conjecture is true.

Conjecture 2.7 $\mathfrak{P}_{1} \neq \mathfrak{P}_{2}$, i.e., the class of central segment orders of the first kind and the class of central segment orders of the second kind are distinct. Furthermore, $\mathfrak{P}_{1} \nsubseteq \mathfrak{P}_{2}$ and $\mathfrak{P}_{2} \nsubseteq \mathfrak{P}_{1}$.

However, we believe (but are not as certain) that the following may be true.

Conjecture 2.8 $\mathfrak{p}_{1}=\mathfrak{P}_{1}$ and $\mathfrak{p}_{2}=\mathfrak{P}_{2}$, i.e., every segment order of the first kind is also a central segment order of the first kind, and every segment order of the second kind is also a central segment order of the second kind.

To support our main conjecture, we will prove a statement which says that there is no continuous function mapping regular line segments to line segments that turns 
each $\mathfrak{P}_{1}$ order into a $\mathfrak{P}_{2}$ order. This statement has an important corollary which states that there is no continuous shift insensitive universal function between $\mathfrak{p}_{1}$ and $\mathfrak{p}_{2}$ (rigorous definitions will be provided later).

Since our ultimate goal is to prove $\mathfrak{P}_{1} \neq \mathfrak{P}_{2}$, the reader might think that eventually someone will construct a $\mathfrak{P}_{1}$ poset with less than a hundred line segments and show that it is not in $\mathfrak{P}_{2}$. While we cannot rule out this possibility completely, the discussion to follow will show that it is very improbable. It seems to us that a counterexample (a poset belonging to one class but not the other) will be extremely large and will require a very complicated argument to establish this fact.

\section{Basic Properties of Segment Orders}

Theorem 3.1 Every poset of dimension at most 3 is in both $\mathfrak{p}_{1}$ and $\mathfrak{p}_{2}$.

Proof Let $P$ be a poset with $\operatorname{dim}(P) \leq 3$. Then let $L_{1}, L_{2}$ and $L_{3}$ be linear orders on the ground set of $P$ such that $u<v$ in $P$ if and only if $u<v$ in $L_{i}$ for each $i=1,2,3$. Let $n$ be the number of elements in $P$ and set $\varepsilon=1 / 10 n$.

For each $u \in P$, let $u_{i}$ be the position of $u$ in $L_{i}$, counting from low to high. For each $u \in P$, let $S(u)$ be the regular line segment satisfying: (1) the left endpoint of $S(u)$ is at $\left(-u_{1}, 0\right)$; (2) $S(u)$ passes through $\left(0, u_{2}\right)$; and (3) the $x$-coordinate of the right endpoint of $S(u)$ is $\varepsilon u_{3}$. It is easy to see $S$ is a representation of $P$ as a segment order of the first kind.

To prove that $P$ also belong to $\mathfrak{p}_{2}$, replace the third condition with $\left(3^{\prime}\right)$ the $x$ coordinate of the right endpoint of $S(u)$ is $\varepsilon\left(n+1-u_{3}\right)$.

Proposition 3.2 For every positive integer $n \geq 3, S_{n} \in \mathfrak{p}_{1}$ and $S_{n} \in \mathfrak{p}_{2}$.

Proof Let $C$ be the quarter circle whose equation is $(x-1)^{2}+y^{2}=4$ with $x \leq 1$ and $y \geq 0$. Let $p_{1}, p_{2}, \ldots, p_{n}$ be the points on $C$, such that the $x$-coordinate of $p_{i}$ is $i /(n+1)$. For each $i=1, \ldots, n$, let $a_{i}$ be a line segment whose left endpoint is of the form $(x, 0)$ for some $-1<x<0$ and right endpoint is $p_{i}$. Let $b_{i}$ be a segment of the tangent of $C$ at point $p_{i}$, such that its left endpoint lies on the $x$ axis and its right endpoint has $x$-coordinate 2 . Then $\left\{a_{1}, \ldots, a_{n}, b_{1}, \ldots, b_{n}\right\}$ is a $\mathfrak{p}_{1}$ representation of $S_{n}$, hence $S_{n} \in \mathfrak{p}_{1}$. (See Fig. 1.)

Showing that $S_{n} \in \mathfrak{p}_{2}$ is very similar, but the choice of $C$ is slightly more complicated. Let $C$ be the arc $y=e^{x}$ with $x \in[0,1]$. Let $p_{1}, p_{2}, \ldots, p_{n}$ be the points on $C$, such that the $x$-coordinate of $p_{i}$ is $i /(n+1)$. For each $i=1, \ldots, n$, let $a_{i}$ be a line segment whose left endpoint is of the form $(x, 0)$ with $x<-1$ and right endpoint is $p_{i}$. Let $b_{i}$ be a segment of the tangent of $C$ at point $p_{i}$, such that its left endpoint lies on the $x$-axis and its right endpoint has $x$-coordinate 2 . Then $\left\{a_{1}, \ldots, a_{n}, b_{1}, \ldots, b_{n}\right\}$ is a $\mathfrak{p}_{2}$-representation of $S_{n}$, hence $S_{n} \in \mathfrak{p}_{2}$. (See Fig. 2.)

On the other hand, there are many posets that are not segment orders. In fact, almost all posets that have dimension at least 4 belong to none of the classes of segment orders we have discussed. The following theorem is a straightforward consequence of the Alon-Scheinerman "degrees of freedom" technique [1]. 


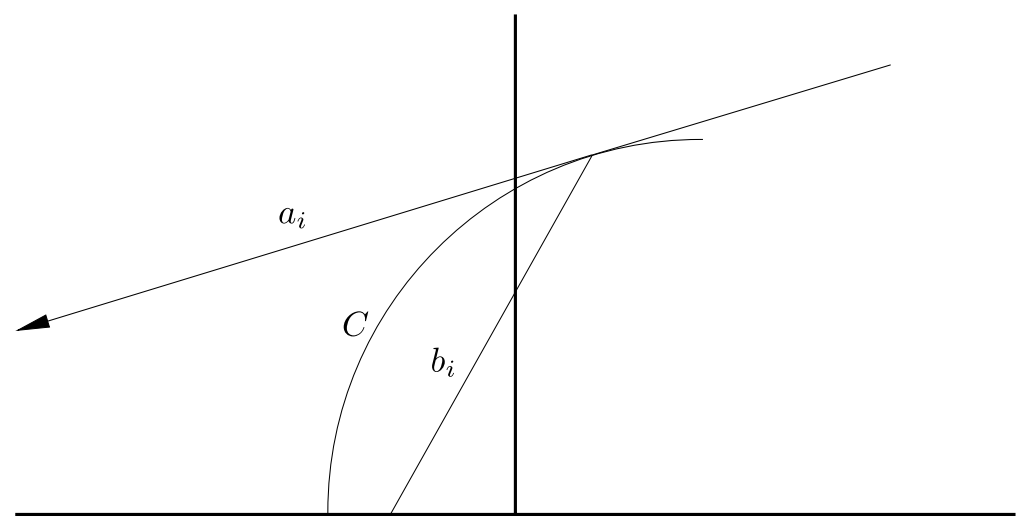

Fig. $1 \mathfrak{p}_{1}$-representation of $S_{n}$

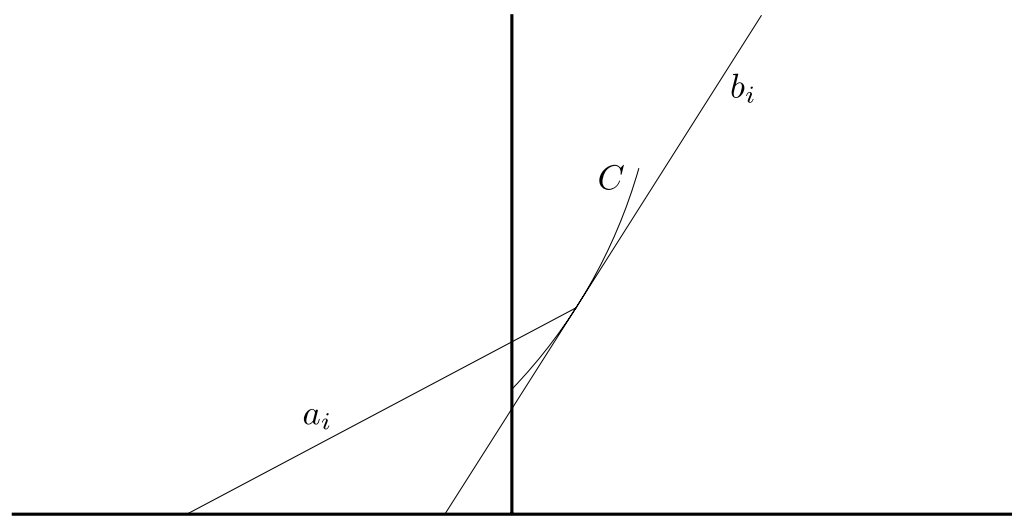

Fig. $2 \mathfrak{p}_{2}$-representation of $S_{n}$

Theorem 3.3 For every $d \geq 4$ and every $\varepsilon>0$, there is an integer $n_{0}$ so that if $n \geq n_{0}$ then among the family of all posets having ground set $\{1,2, \ldots, n\}$ the ratio of the number of posets that belong to $\mathfrak{p}_{1} \cup \mathfrak{p}_{2}$ divided by the total number of posets is less than $\varepsilon$.

Let $n \geq 3$ and let $S_{n}$ be the standard example. Note that the subposet determined by $\left\{a_{1}, a_{2}, b_{1}, b_{2}\right\}$ is the union of two 2-element chains, with points from one chain incomparable with points in the other. This poset is frequently denoted $\mathbf{2}+\mathbf{2}$. As is well known, this poset characterizes interval orders. The following result is usually credited to Fishburn [4] who was the first researcher to state and prove the result explicitly.

Theorem 3.4 A poset $P$ is an interval order if and only if it does not contain $2+2$ as a subposet.

So the fact that large standard examples belong to $\mathfrak{p}_{1}$ and $\mathfrak{p}_{2}$ says nothing about interval orders. Also, we noted earlier that all interval orders belonged to $\mathfrak{P}_{2}$. Now 


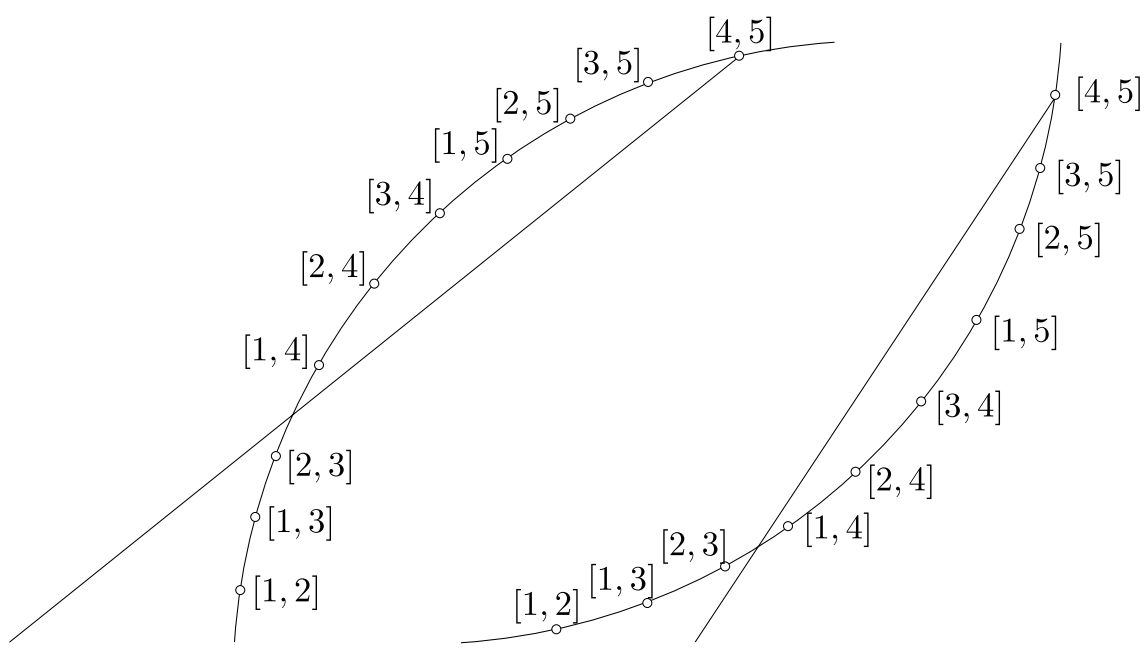

Fig. 3 Right end points of segments and the segment assigned to [4, 5]

we show more. The following proof is using ideas similar to those that are presented in [5].

Theorem 3.5 Every interval order is in both $\mathfrak{p}_{1}$ and $\mathfrak{p}_{2}$.

Proof It is clearly sufficient to prove that the canonical interval order $I_{n}$ belongs to $\mathfrak{p}_{1} \cap \mathfrak{p}_{2}$ for each $n \geq 2$. We first show that $I_{n} \in \mathfrak{p}_{1}$.

Draw a quarter of a circle with radius 1 and center $(1,0)$, as shown on the left of Fig. 3. For every interval of $I_{n}$, place a point to the circle, in the way it is shown in the figure. For every point $[i, j]$, if $i \geq 3$, draw a half-line from the point representing $[i, j]$ so that it intersects the circle between the point representing $[i-2, i-1]$ and the next point up on the circle. Drop the portion of this half-line that is under the $x$-axis to create a line segment. This line segment will be assigned to the interval $[i, j]$.

If $i \leq 2$, draw a line segment from $(0, \varepsilon)$ to the point representing $[i, j]$, where $\varepsilon$ is a small positive real, such that $(0, \varepsilon)$ will be the point closest to the origin on the $x$-axis of all the end points of the segments.

It is clear that the $\mathfrak{p}_{1}$ ordering among these segments is the same as the interval ordering among the intervals.

If we change the quarter circle as it is shown on the right of Fig. 3, then the same argument gives a proof that every interval order is a $\mathfrak{p}_{2}$ order.

\section{Further Goals and Motivations}

We have not been able to find a $\mathfrak{p}_{1}$ poset that is not $\mathfrak{p}_{2}$ or vice versa. The previous discussion shows that if there is one, it is of dimension at least 4 , not an interval order 
and not a standard example. If the two classes are the same, one would expect that there is short proof, which would be something like a nice, bijective function from the regular line segments of $\mathbb{R}^{2}$ to itself that maps the line segments of a $\mathfrak{p}_{1}$ poset to another set of line segments, so that it will be a $\mathfrak{p}_{2}$-representation of the same poset. Or maybe its is even true for $\mathfrak{P}_{1}$ and $\mathfrak{P}_{2}$. Momentarily, we will show, that at least the latter statement is false.

From the remainder of the paper, we focus on the question as to whether $\mathfrak{p}_{1}=\mathfrak{p}_{2}$.

Definition 4.1 A universal function is a function $f: \mathbb{R}^{3} \rightarrow \mathbb{R}^{3}$ such that

(1) For every regular segment $s=\left(s_{1}, s_{2}, s_{3}\right)$, the image $f(s)=t=\left(t_{1}, t_{2}, t_{3}\right)$ is a regular segment; and

(2) If $s$ and $t$ are regular line segments, then $s>t$ in $\mathfrak{P}_{1}$ if and only if $f(s)>f(t)$ in $\mathfrak{P}_{2}$.

When $s=\left(s_{1}, s_{2}, s_{3}\right)$ is a regular line segment and $c$ is a real number, we let $s+c$ denote the regular line segment $\left(s_{1}+c, s_{2}+c, s_{3}\right)$. Note that $s+c$ is just a horizontal translation (shift) of $s$.

Definition 4.2 A universal function $f: \mathbb{R}^{3} \rightarrow \mathbb{R}^{3}$ is shift insensitive if for every real number $c$, there exists a real number $c^{\prime}$ so that $f(s+c)=f(s)+c^{\prime}$ for every regular segment $s$.

The partial result that we have obtained is the following.

Theorem 4.3 There is no continuous shift insensitive universal function.

\section{Assuming the Two Classes Are the Same}

Throughout this section, we will be assuming that every finite segment order of the first kind is also a segment order of the second kind—even though this is something we do not believe to be true. Accordingly, we will consider $\mathfrak{P}_{1}$ posets whose elements are regular line segments in a representation of the first kind and that the segments are in a general position. For an element $s=\left(s_{1}, s_{2}, s_{3}\right)$ of $P$, we will then let $\left(s_{1}^{\prime}, s_{2}^{\prime}, s_{3}^{\prime}\right)$ be the coordinates of the regular segment corresponding to $s$ in a representation of $P$ as a segment order of the second kind, with the segments again in a general position. We will then derive some additional restrictions that we may assume are satisfied by representation of $P$ as a segment order of the second kind. Of course, the end goal is to gather sufficiently many restrictions to force a contradiction.

\subsection{End Point Extensions}

Definition 5.1 Let $P$ be a poset in $\mathfrak{p}_{i}$ with a representation $P_{i}$. We say, $L$ is the left linear extension of $P$ (defined by $P_{i}$ ), if $L$ is defined by the regular ordering of the absolute values of the $x$-coordinates of the left endpoints of the line segments of $P_{i}$. Similarly, we say, $R$ is the right linear extension of $P$ (defined by $P_{i}$ ), if $R$ 
is defined by the regular ordering of the absolute values of the $x$-coordinates of the right endpoints of the line segments of $P_{i}$.

Let $L_{1}$ be the left linear extension of $P$ (defined by $P_{1}$ ), and let $R_{1}$ be the right linear extension of $P$ (defined by $P_{1}$ ). Similarly, let $L_{2}$ be the left linear extension of $P$ (defined by $P_{2}$ ), and let $R_{2}$ be the right linear extension of $P$ (defined by $P_{2}$ ).

Lemma 5.2 We may assume that $L_{2}=R_{1}$ and that $R_{2}=L_{1}^{d}$ (the dual of $L_{1}$ ).

Proof To prove the lemma, we will first consider the poset $P$ as a subposet of a much larger poset $Q$ which also belongs to $\mathfrak{P}_{1}$. We will then take a representation of $Q$ as a segment order of the second kind and show that the segments corresponding to elements of $P$ must be properly positioned.

The construction of $Q$ comes in two parts, i.e., we first put $P$ inside $Q_{1}$ and then put $Q_{1}$ inside an even larger poset $Q_{2}=Q$. With the first extension, we force an extension in which $L_{2}=R_{1}$. Then with the second extension, we force $R_{2}$ to be the dual of $L_{1}$.

\subsection{First Part}

Let $n=|P|$. Consider a large positive $m$ (later, it will be clear just how large $m$ be must be relative to $n$ ). We will add to $P$ segments that form a copy of a standard example $S_{m}$ of dimension $m$. These segments will be denoted $A=\left\{a_{1}, a_{2}, \ldots, a_{m}\right\}$ and $B=\left\{b_{1}, b_{2}, \ldots, b_{m}\right\}$ with $a_{i}>b_{j}$ if and only if $i \neq j$. We restrict the position of segments further as shown in Fig. 1. The elements of $B$ are confined to a narrow space: each of them starts after the last element of $P$ (i.e., the left endpoints of the elements of $P$ are left from the left endpoints of the elements of $B$ ), and each of the ends before the first ending element of $P$. Also, $p_{i} \cap a_{j}=\emptyset$ for all $i, j$.

Additionally, we construct a set of line segments $G$.

$$
G=\left\{g_{S}: S \text { is a set of consecutive integers not greater than } n\right\} .
$$

We define $G$ so that $G \| A$, and for each $S$ for which $g_{S} \in G$, it holds that $g_{S} \|\{b \in$ $B: b \in S\}$ but $g_{S}>\{b \in B: b \notin S\}$. Note, that this does not define how to draw the line segments of $G$, but clearly, it is easy to do it ("intermix" the segments with the elements of $A$ ). (See Fig. 4.)

Suppose that the $x$-coordinates of the right endpoints of the elements of $p_{1}, \ldots, p_{|P|}$ are $p_{1}^{\mathrm{R}}, \ldots, p_{|P|}^{\mathrm{R}}$. Without loss of generality, $p_{1}^{\mathrm{R}}<\cdots<p_{|P|}^{\mathrm{R}}$. Note, this is equivalent to the statement $R_{1}=\left(p_{1}, \ldots, p_{n}\right)$. Then the $x$-coordinates of the right endpoints of the elements of $A$, call them $a_{1}^{\mathrm{R}}, \ldots, a_{n}^{\mathrm{R}}$ will follow the following rule:

$$
a_{i}^{\mathrm{R}}=\frac{p_{j}^{\mathrm{R}}+p_{j+1}^{\mathrm{R}}}{2} \quad \text { if } i=\left(k-\frac{1}{2}\right) 2^{j} \text { for some } k \text { integer. }
$$

Some $a_{i}^{\mathrm{R}}$ are not defined by this rule (namely, iff $i \mid 2^{|P|}$ ); they will be such that $a_{i}^{\mathrm{R}}>p_{j}^{\mathrm{R}}$ for all $j$, but otherwise arbitrary. 


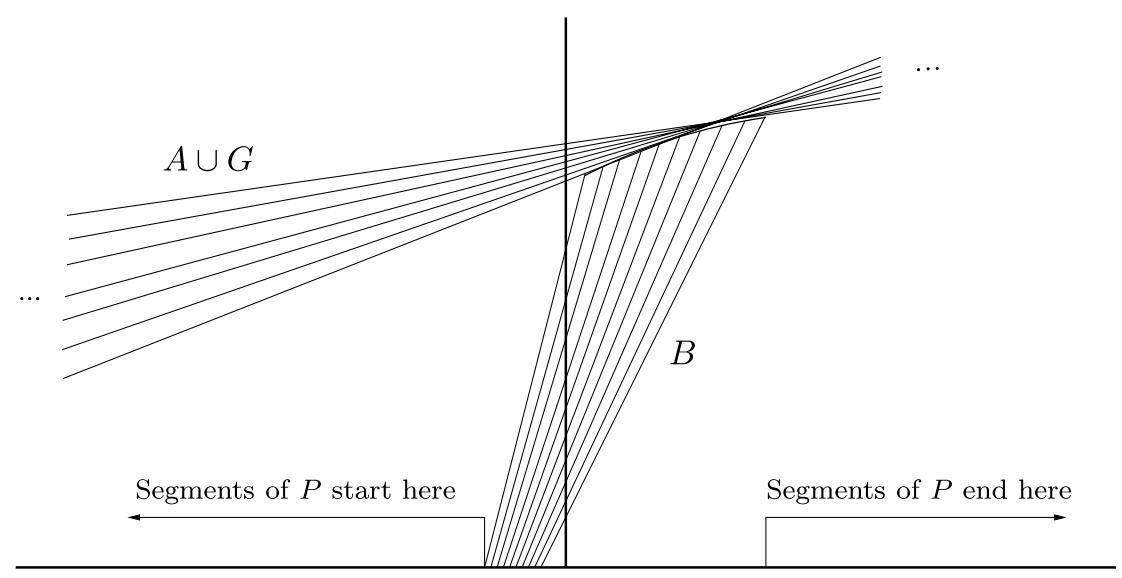

Fig. 4 Arrangement of $Q_{1}$

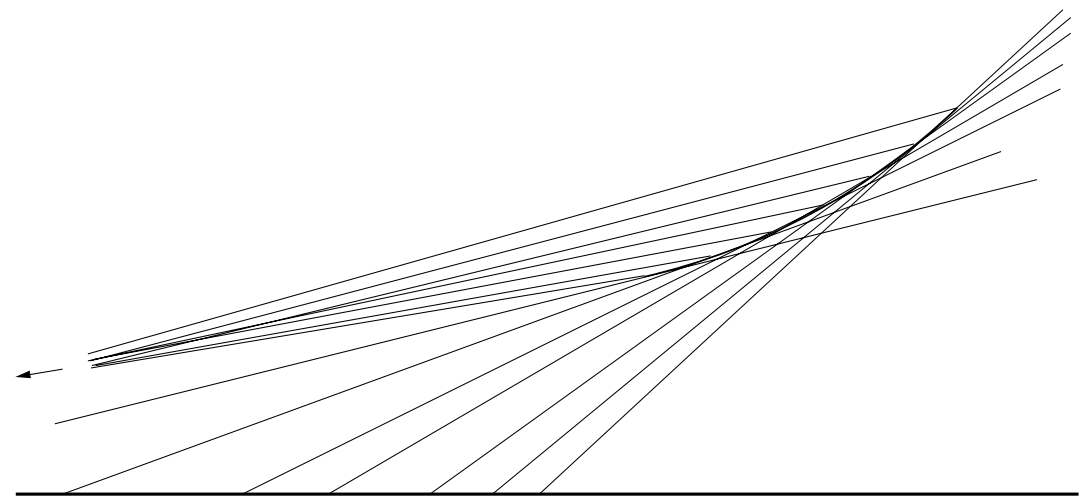

Fig. $5 \mathfrak{p}_{2}$-representation of $S_{n}$

Now we will show that $Q_{2}$ has to attain a very specific form, and so does $P_{2}$. From now on, we change the notation, and $p_{i}, a_{i}, b_{i}$ denote line segments of the $\mathfrak{p}_{2}$-representation, and we use again $a_{i}^{\mathrm{L}}, a_{i}^{\mathrm{R}}$ for the absolute values of the $x$ coordinates of the left and right endpoints of the segments. However, we maintain the indices, so that in this way $a_{i}$ is the image of the original $a_{i}$. (We believe that the confusion this might cause is less than the confusion that would be caused by the hordes of indices.)

In the following, we will study the structure of $A \cup B$ in the $\mathfrak{p}_{2}$-representation. Since this will be useful later, too, we state it as a separate lemma.

Lemma 5.3 Every $\mathfrak{p}_{2}$-representation of $S_{n}$ (standard example) has the arrangement as in Fig. 5, with the exception of at most two pairs of line segments. 
Proof The sets $A$ and $B$ are the maximum antichains in $S_{n} . A=\left\{a_{1}, \ldots, a_{n}\right\}, B=$ $\left\{b_{1}, \ldots, b_{n}\right\}$ and $a_{i}>b_{j}$ if and only if $i \neq j$.

Hence in the representation,

$$
a_{i}^{\mathrm{L}}>b_{j}^{\mathrm{L}}
$$

for all $i, j$ except maybe for one pair. Let us ignore that pair now and concentrate on the remaining. Now let $a_{k}$ be such that $a_{k}^{\mathrm{R}}$ is maximal. Then each $b_{i}<a_{k}$ with $i \neq k$, so each $b_{i}^{\mathrm{R}}>a_{k}^{\mathrm{R}}$ with $i \neq k$. If we ignore $a_{k}$ and $b_{k}$ now, too, then the remaining

$$
\left\{a_{i}^{\mathrm{R}}<b_{j}^{\mathrm{R}}\right\} \quad \text { for all } i, j, \text { except for the ignored. }
$$

Still, for every $i, a_{i} \| b_{i}$ somehow: the only way this can be is if $a_{i} \cap b_{i} \neq \emptyset$. So the $\mathfrak{p}_{2}$-representation must look like Fig. 5 .

We can specify an ordering on $B$ with the aid of the left linear extension (defined by $\left.Q_{2}\right)$, call it $\left.L_{2}\left(Q_{2}\right)\right|_{B}$. Also, denote the linear order specified by the left linear extension (defined by $Q_{1}$ ) by $\left.L_{1}\left(Q_{1}\right)\right|_{B}$. We claim that if we keep ignoring the two pairs in the previous paragraph, then either

$$
\left.L_{1}\left(Q_{1}\right)\right|_{B}=\left.L_{2}\left(Q_{2}\right)\right|_{B} \quad \text { or }\left.\quad L_{1}\left(Q_{1}\right)\right|_{B}=\left(\left.L_{2}\left(Q_{2}\right)\right|_{B}\right)^{d} .
$$

To see this, pick $b_{l}$ and $b_{m}$ consecutive elements in $\left.L_{1}\left(Q_{1}\right)\right|_{B}$. We know that $m=l+1$ unless some elements were ignored between them, in which case every $i: l<i<m$ is an index of an ignored element. Let $S=\{l, \ldots, m\}$ a set of (at least two) integers, and consider the image of $g_{S}$, call it $g^{*}$. Now $g^{*}>b_{i}$ for all $i$ except for the images of $b_{l}$ and $b_{m}$ (and the ignored elements, of course). This is only possible if the images of $b_{l}$ and $b_{m}$ are consecutive line segments in Fig. 5, or more rigorously, the images of $b_{l}$ and $b_{m}$ are consecutive in $\left.L_{2}\left(Q_{2}\right)\right|_{B}$. So we deduced that the consecutivity property is preserved between $\left.L_{1}\left(Q_{1}\right)\right|_{B}$ and $\left.L_{2}\left(Q_{2}\right)\right|_{B}$, which implies (2). (In fact, this is all we need the set $G$ for.)

Note that the statement above has no consequence to the right linear extension of $B$. However, it immediately implies a similar statement on the right linear extension of $A$. Let $\left.L_{2}\left(Q_{2}\right)\right|_{A}$ be the right linear extension (defined by $Q_{2}$ ). Then

$$
\left.L_{2}\left(Q_{2}\right)\right|_{A}=\left(a_{1}, \ldots, a_{n}\right) \quad \text { or } \quad\left(a_{n}, \ldots, a_{1}\right) .
$$

(Again, recall that we may have missing elements from these sets, but that will not affect our argument.)

Recall that $R_{1}=\left(p_{1}, \ldots, p_{|P|}\right)$, so we need to show that $L_{2}=\left(p_{1}, \ldots, p_{|P|}\right)$.

Now recall the definition of $Q_{1}$, specifically that all $a_{i}$ had its left endpoint left from all $p_{i}$, and the ordering of their right endpoints by (1). In particular, it implies that $p_{1}<a_{i}$ for all $i$. Therefore, it must be that $p_{1}<_{L_{2}} a_{i}$ for all $i$. Also from (1), $p_{2}<a_{i}$ if and only if $i$ is even, and $p_{2} \| a_{i}$ if and only if $i$ is odd. Therefore, using (3), the only possible arrangements for $A$ in $Q_{2}$ is that the even indexed left endpoints are grouped together, and also the odd indexed left endpoints are grouped together, and $p_{2}$ 's left endpoint is between the two groups. In particular, $p_{1}<_{L_{2}} p_{2}$. (See Fig. 6.) 


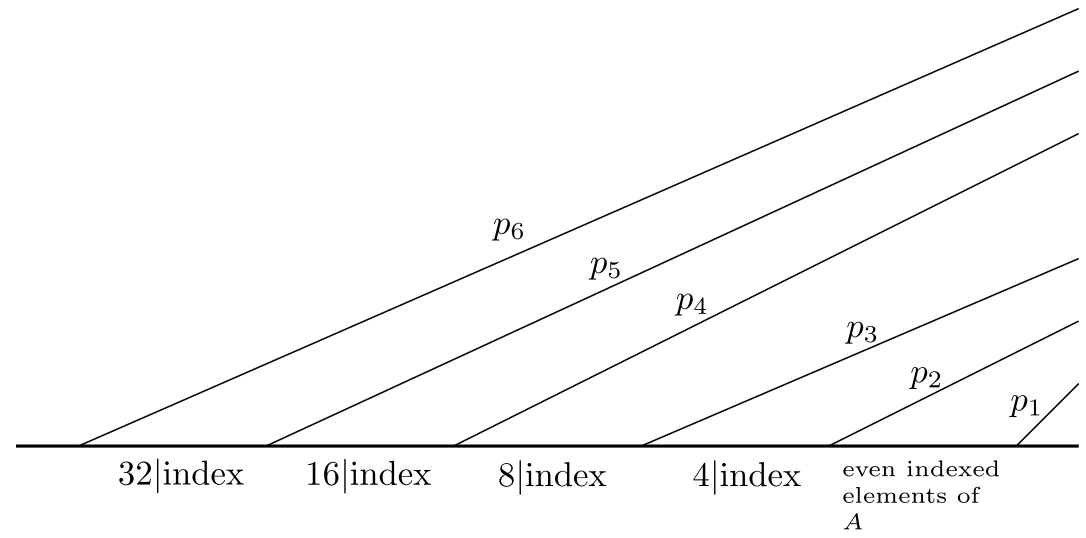

Fig. 6 Left endpoints of $A$ and $P$ in the $\mathfrak{p}_{2}$-representation

We can continue this argument by considering $p_{3}, p_{4}$, and so on, in every step concluding, that the given $p_{j}<a_{i}$ if and only if $i \mid 2^{j-1}$, otherwise $p_{j} \| a_{i}$. So the left endpoints of $A$ are grouped in a manner shown in Fig. 6, and the left endpoints of $P$ must be between the groups. That forces an order on the left endpoints of $P$, namely

$$
p_{1}<_{L_{2}} p_{2}<_{L_{2}} \cdots<_{L_{2}} p_{|P|} \text {. }
$$

As a last remark, let us add that we needed all $2^{|P|+1}$ elements of $A$ and $B$. If we did not have to "ignore" elements early in the argument, then $2^{|P|-1}$ would have been enough, but in the current situation, we had to defend us against the situation when crucial elements, that would force ordering on $P$, are ignored. Using $2^{|P|+1}$ intervals ensures that there are at least 4 elements of $A$ in every important group on Fig. 6 (3 would have been enough, but it is just simpler to increase the exponent of two by 1 ), so even if we ignore 2 of those, at least 1 is left to do its job.

\subsection{Second Part}

Surprisingly, repeating the techniques of the first part of the proof does not seem to work. The ideas are somewhat simpler here, but the description of the proof is more technical, and we heavily use the first part of the lemma.

As we mentioned earlier, in this part of the proof, we will assume that $Q_{1}$ and $Q_{2}$ already obey the first part of the lemma.

For every pair of incomparable line segments in $P_{1}$, we will add some extra line segments to form a portion of $Q_{1}$. The configuration of the new line segments depends on the configuration of the two original line segments. According to this, we will separate several cases.

In the following cases, the two line incomparable segments will always be denoted by $r$ and $b$, and always so that the left endpoint of $r$ is left from the left endpoint of $b$ in $P_{1}$. We will also use the notation $a^{\mathrm{L}}, a^{\mathrm{R}}$ for a line segment $a$ as in the previous section, and we will add the notation $a^{\mathrm{U}}$ for the $y$-coordinate of the right endpoint 

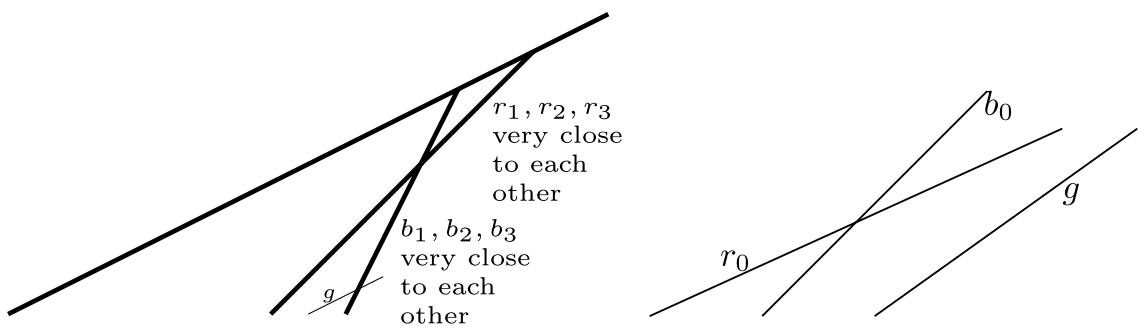

Fig. 7 Case 1

of $a$. Also, we keep the convention that we will use the same letter for the line segment in $Q_{2}$ (or specially $P_{2}$ ) as for the line segment in $Q_{1}$ (or $P_{1}$ ) and we will make it clear with the context which line segment is the argument about.

Using these notation and assumptions, in every case we will prove that

$$
r^{\mathrm{L}}>b^{\mathrm{L}} \quad \text { in } P_{1} \Longrightarrow r_{0}^{\mathrm{R}}<b_{0}^{\mathrm{R}} \quad \text { in } P_{2},
$$

where most of the time $r_{0}=r$ and $b_{0}=b$, and other times $r_{0} \neq r$, but $r_{0}$ has the same relation to any other element of $P_{1}$ as $r$, and similar statement is true for $b$.

Since we eventually build helpers above every pair of incomparable line segments, this statement is going to be proven for every pair of incomparable line segments, and therefore the second part of the lemma follows.

\subsubsection{Case 1 (Fig. 7)}

$$
b^{\mathrm{R}}<r^{\mathrm{R}} \quad \text { and } \quad b^{\mathrm{U}}<r^{\mathrm{U}} \quad \text { in } Q_{1} .
$$

Relabel $r$ to $r_{1}$ and $b$ to $b_{1}$, and add extra line segments $b_{2}, b_{3}, r_{2}, r_{3}$ so that $b_{1}^{\mathrm{R}}<$ $b_{2}^{\mathrm{R}}<b_{3}^{\mathrm{R}}<r_{1}^{\mathrm{R}}<r_{2}^{\mathrm{R}}<r_{3}^{\mathrm{R}}$ and $b_{1}^{\mathrm{U}}<b_{2}^{\mathrm{U}}<b_{3}^{\mathrm{U}}<r_{1}^{\mathrm{U}}<r_{2}^{\mathrm{U}}<r_{3}^{\mathrm{U}}$ and $b_{1}^{\mathrm{L}}<b_{2}^{\mathrm{L}}<b_{3}^{\mathrm{L}}<$ $r_{1}^{\mathrm{L}}<r_{2}^{\mathrm{L}}<r_{3}^{\mathrm{L}}$, and make sure that $b_{2}$ and $b_{3}$ have the same relation to every element of the poset as $b_{1}$, similarly for $r_{2}, r_{3}$ and $r_{1}$.

Add 6 additional segments so that they now form $S_{6}$. Also add the line segment $g$ so that $b_{3}^{\mathrm{L}}<g^{\mathrm{L}}<r_{1}^{\mathrm{L}}$ and $g^{\mathrm{R}}<b_{1}^{\mathrm{R}}$. Also $g<Q r_{i}$ for $i=1,2,3$, and $g$ intersects $b_{i}$ for $i=1,2,3$ in $Q_{1}$.

Apply Lemma 5.3 to the poset induced by $r_{1}, r_{2}, r_{3}$ and $b_{1}, b_{2}, b_{3}$. In $Q_{2}$, there must be a pair of segments, call them $r_{0}$ and $b_{0}$, that intersect. Where is $g$ in $Q_{2}$ ? Due to the first part of the lemma, $g^{\mathrm{L}}<b_{0}^{\mathrm{L}}<r_{0}^{\mathrm{L}}$. Suppose that $b_{0}^{\mathrm{R}}<r_{0}^{\mathrm{R}}$; then, due to $g<Q r_{0}, g$ cannot possibly intersect $b_{0}$ in $Q_{2}$, so $g<_{Q} b_{0}$, a contradiction. We showed that $r_{0}^{\mathrm{R}}<b_{0}^{\mathrm{R}}$. (This is the only part of the proof where $r$ may not be identical to $r_{0}$ and $b$ may not be identical to $b_{0}$.)

\subsubsection{Case 2 (Fig. 8)}

$$
b^{\mathrm{R}}<r^{\mathrm{R}} \text { and } \quad r^{\mathrm{U}}<b^{\mathrm{U}} \text { in } Q_{1} .
$$




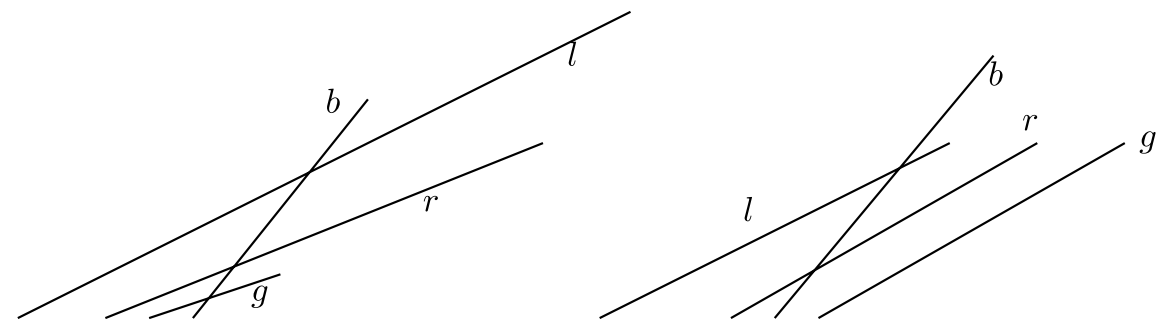

Fig. 8 Case 2
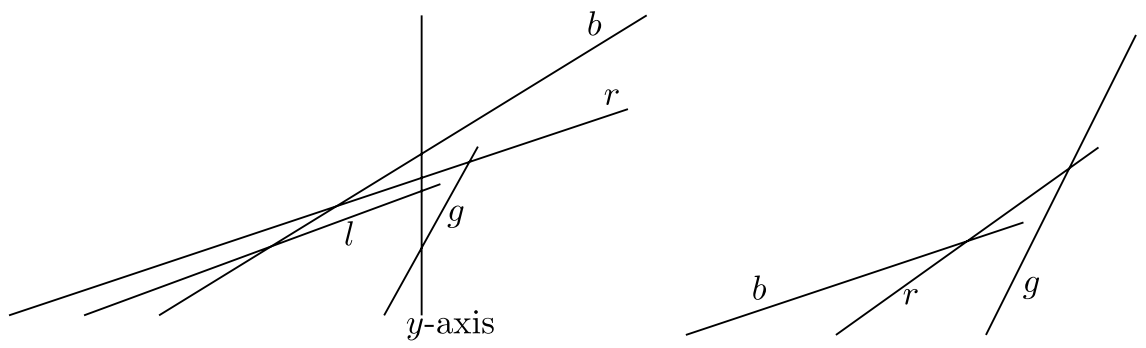

Fig. 9 Case 3

We need to add two extra line segments, $g$ and $l$, so that $b^{\mathrm{L}}<g^{\mathrm{L}}<r^{\mathrm{L}}<l^{\mathrm{L}}, g^{\mathrm{R}}<$ $b^{\mathrm{R}}<r^{\mathrm{R}}<l^{\mathrm{R}}, g^{\mathrm{U}}<r^{\mathrm{U}}<b^{\mathrm{U}}<l^{\mathrm{U}}$ in $Q_{1}$. By the first part, $g^{\mathrm{L}}<b^{\mathrm{L}}<r^{\mathrm{L}}<l^{\mathrm{L}}$ in $Q_{2}$. Also $g<_{Q} r<_{Q} l$. By Case 1 , we may assume that $b$ intersects $l$. If $b^{\mathrm{R}}<r^{\mathrm{R}}$, then $b>g$, a contradiction. So $r^{\mathrm{R}}<b^{\mathrm{R}}$.

\subsubsection{Case 3 (Fig. 9)}

$$
r^{\mathrm{R}}<b^{\mathrm{R}} \quad \text { and } \quad r \cap b \neq \emptyset \quad \text { in } Q_{1} \text {. }
$$

Add two line segments, $g$ and $l$, so that in $Q_{1}$

- $g<_{Q} b$

- $g \|_{Q} r$, and $g$ and $r$ are in the same relation as $b$ and $r$ in Case 1

- $g^{\mathrm{R}}<r^{\mathrm{R}}$ and $g^{\mathrm{R}}<b^{\mathrm{R}}$

- $l<Q^{r}$

- $l \|_{Q} b$

- $l^{\mathrm{R}}$ is less than all of $g^{\mathrm{R}}, r^{\mathrm{R}}, b^{\mathrm{R}}$

In $Q_{2}, b^{\mathrm{L}}>r^{\mathrm{L}}$ by the first part of the lemma. Suppose that $b^{\mathrm{R}}<r^{\mathrm{R}}$. Then $b$ and $r$ must intersect in $Q_{2}$ in order to be incomparable. We know that $g^{\mathrm{L}}<r^{\mathrm{L}}, g<_{Q} b$ and $g \|_{Q} r$. Also, $g \cap r \neq \emptyset$ and $g^{\mathrm{R}}>r^{\mathrm{R}}$ by the Case 1 construction. Then it should happen that $l<Q r, l \|_{Q} b$ and $l^{\mathrm{L}}<g^{\mathrm{L}}$. This is impossible, so $r^{\mathrm{R}}<b^{\mathrm{R}}$. 
Fig. 10 Case 4

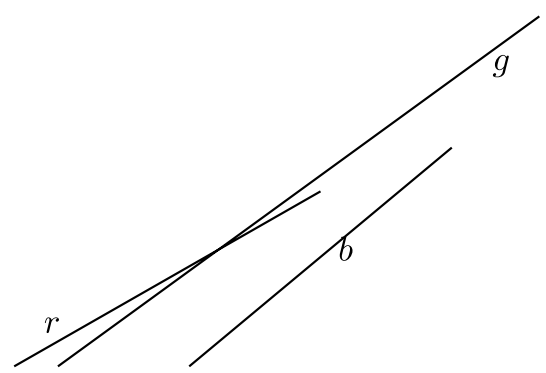

5.3.4 Case 4 (Fig. 10)

$$
r^{\mathrm{R}}<b^{\mathrm{R}} \quad \text { and } \quad r \cap b=\emptyset \quad \text { in } Q_{1} \text {. }
$$

Add a line segment $g$ such that $g>_{Q} b$ and $g$ and $r$ are in the same relation as $b$ and $r$ in Case 3. In $Q_{2}, g^{\mathrm{R}}<b^{\mathrm{R}}$, because $g>_{Q} b$. If $b^{\mathrm{R}}<r^{\mathrm{R}}$, then $g^{\mathrm{R}}<r^{\mathrm{R}}$, contradicting Case 3.

This concludes the proof of the main lemma.

Although we worked out the proof for $\mathfrak{p}_{1}$ and $\mathfrak{p}_{2}$, observe that position of the $y$-axis did not play a crucial role in the proof. By dropping the assumption that every line segment intersects the $y$-axis, the proof still stands. Therefore, we can also conclude the following:

Lemma 5.4 The statement of Lemma 5.2 holds for $\mathfrak{P}_{1}$ and $\mathfrak{P}_{2}$.

\section{Connections with Pseudoline Arrangements}

In this section, unless otherwise noted, we will work on the real projective plane $\mathbb{P}^{2}$.

Definition 6.1 A pseudoline is a simple closed curve whose removal does not disconnect the plane. An arrangement of pseudolines is a set of pseudolines such that any two intersects at exactly one point, and not all of them intersect in the same point. Two pseudoline arrangements are isomorphic, if there is a homeomorphism that maps one to the other. A pseudoline arrangement is stretchable if it is isomorphic to a pseudoline arrangement in which every pseudoline is a straight line.

Not every pseudoline arrangement is stretchable. To show a counterexample, recall the classical geometrical theorem by Pappus.

Theorem 6.2 (Pappus) Let $a_{1}, a_{2}, a_{3}$ be collinear points, and $b_{1}, b_{2}, b_{3}$ be another set of collinear points. Let $\left(a_{i}, b_{j}\right)$ denote the straight line that passes through the points $a_{i}$ and $b_{j}$. Then the points $\left(a_{1}, b_{2}\right) \cap\left(a_{2}, b_{1}\right),\left(a_{1}, b_{3}\right) \cap\left(a_{3}, b_{1}\right),\left(a_{2}, b_{3}\right) \cap$ $\left(a_{3}, b_{2}\right)$ are collinear.

Corollary 6.3 The pseudoline arrangement on Fig. 11 is not stretchable. 


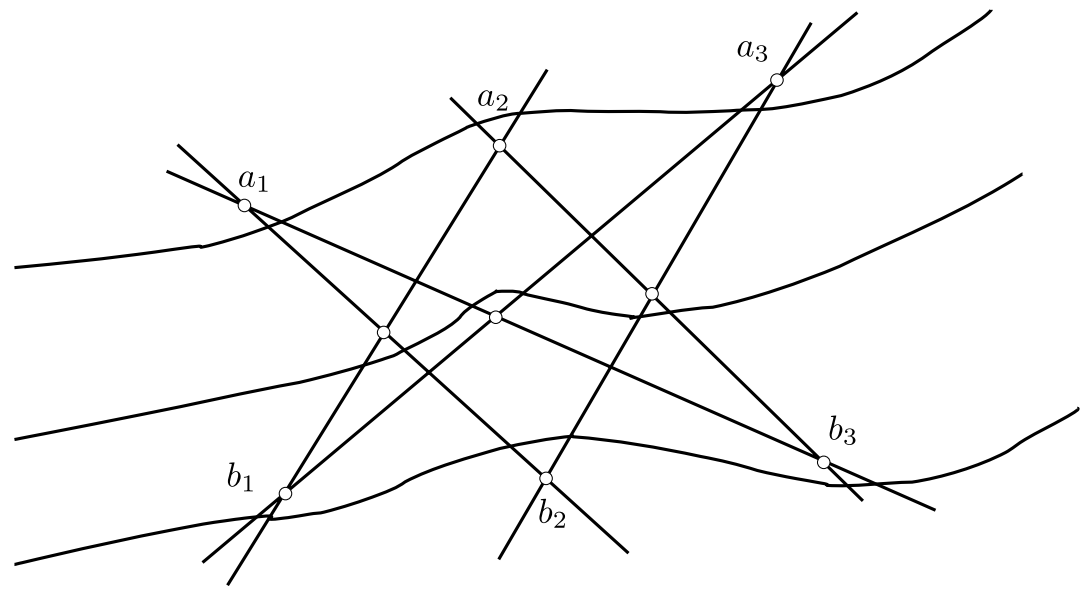

Fig. 11 Non-stretchable simple arrangement with 9 pseudolines

Desargues' Theorem (see [3]) can be used to produce a non-stretchable configuration on 10 lines. There are several other famous examples. Bokowski and Sturmfels provided a "minor-minimal" infinite family of non-stretchable arrangements. This already suggests that it is difficult to determine if a pseudoline arrangement is stretchable. Indeed, Mnëv [9] (and later Shor [12]) proved that the problem determining if a pseudoline arrangement is stretchable is NP-complete.

Definition 6.4 A pseudoline-arrangement is simple, if no three pseudolines cross at the same point.

For more information on pseudoline arrangements, see [7], or for more detailed exposition from the point of view of oriented matroids, see [2].

In the following, we will see how Conjecture 2.7 is related to stretchability of pseudoline arrangements. We will define a sequence of posets $U_{n} \in \mathfrak{p}_{1}$. We will assign a family of pseudoline arrangements to each $U_{n}$, and we will ask if it is true that there is a stretchable arrangement in each family.

Let $\hat{U}_{n}=\{1, \ldots, n\}^{3}$, the set of ordered triples of positive integers not greater than $n$. To every element $(l, r, u)$ of $\hat{U}_{n}$, assign the line segment from $(-l, 0)$ to $(r, u)$. Consider these line segments as a $\mathfrak{p}_{1}$-representation of a poset. Call this poset $U_{n}$.

If there is an $n$ such that $U_{n} \notin \mathfrak{p}_{2}$, then $\mathfrak{p}_{1} \nsubseteq \mathfrak{p}_{2}$, specifically, Conjecture 2.7 is true. On the other hand, if $U_{n} \in \mathfrak{p}_{2}$ for every $n$ positive integer, then $\mathfrak{p}_{1} \subseteq \mathfrak{p}_{2}$. Indeed, every poset in $\mathfrak{p}_{1}$ can be represented as a subset of $\hat{U}_{n}$ for some large $n$.

The subsets of $\hat{U}_{n}$ that is determined by the triples

$$
P(r, u)=\{(i, r, u): i \in\{1, \ldots, n\}\}
$$

are called pencils. 
We say pencil $P\left(r_{1}, u_{1}\right)$ dominates pencil $P\left(r_{2}, u_{2}\right)$ at level $i$ if

- $\left(r_{2}, u_{2}\right)$ is below the line connecting points $(-i, 0)$ and $\left(r_{1}, u_{1}\right)$, or

- $\left(r_{2}, u_{2}\right)$ is on the line connecting points $(-i, 0)$ and $\left(r_{1}, u_{1}\right)$ and $r_{1}<r_{2}$ and $u_{1}<u_{2}$.

The following proposition is a straightforward consequence of the definition.

Proposition 6.5 The domination relation of pencils at a fixed level $i$ is a total order on the pencils.

In the following, we describe a pseudoline arrangement, that is associated with the poset $U_{n}$. For simplicity, we will use a Cartesian coordinate system, and we add the ideal line. We will only specify ordered sequences of points in the coordinate system that the pseudoline passes through, in the prescribed order. Doing so, we actually do not fully specify the arrangement, but we do specify many (intersection) properties of it. Then we consider all pseudoline arrangements that satisfy these properties to get our family of arrangement.

Start with straight lines $v_{1}, \ldots, v_{n}$ such that $v_{i}$ is the vertical line at $x=n-i+1$. Add a new pseudoline $p_{r, u}$ for each pencil $P(r, u)$. Let $l_{i}(r, u)$ be the position of the pencil $P(r, u)$ at level $i$ in the total order defined by domination, so that $l_{i}(r, u)$ is 1 for the smallest pencil and $n^{2}$ for the largest. Let the pseudoline $p_{r, u}$ pass through $(-r, 0)$, then for $i=1, \ldots, n$, let it pass through $\left(i, l_{i}(r, u)\right)$. (See Fig. 12.)

Observe, that we did not specify how the pseudolines intersect left from $v_{1}$, but it is very strictly defined how they intersect between any $v_{i}$ and $v_{i+1}$. This way, taking every possible arrangement (up to isomorphism), we have defined a family of arrangements. Call it $\mathcal{F}_{n}$.

Theorem 6.6 If there exists an $n \in \mathbb{N}$ such that no pseudoline arrangement in $\mathcal{F}_{n}$ is stretchable, then $\mathfrak{p}_{1} \nsubseteq \mathfrak{p}_{2}$.

Proof Consider $\hat{U}_{n}$, a $\mathfrak{p}_{1}$-representation of $U_{n}$. It is clear that we can break ties with the endpoint coordinates in $\hat{U}_{n}$ without changing the underlying poset. Also, we can move the line segments so that no line segment endpoint lies on another line segment, still without changing the underlying poset.

For each line segment, create two other identical segments, overlapping the original. Now move one replica's left endpoints to left by $\varepsilon$, and move the other's left endpoints to the right by $\varepsilon$. Do this so that the replicas have the same relationship with every other line segments that are not in any copy on this pencil. With carefully done tie breaking it is possible to shift the replicas in such a way, that the negative half of the $x$-axis can be broken into disjoint intervals $I_{1}^{\mathrm{R}}, I_{1}, I_{1}^{\mathrm{L}}, I_{2}^{\mathrm{R}}, \ldots, I_{n}^{\mathrm{L}}$ (in this order going left on the $x$-axis) with the following property: The set of line segments $L_{i}$ whose left end point was $(-i, 0)$ have their left endpoints in $I_{i}$, their leftward shifted replicas have their left endpoints in $I_{i}^{\mathrm{L}}$ and their rightward shifted replicas have their left endpoints in $I_{i}^{\mathrm{R}}$. Call this new $\mathfrak{p}_{1}$-representation $\tilde{U}_{n}$.

We will apply Lemma 5.2 on $\tilde{U}_{n}$; recall that this involves building a huge helper poset above $\tilde{U}_{n}$. 


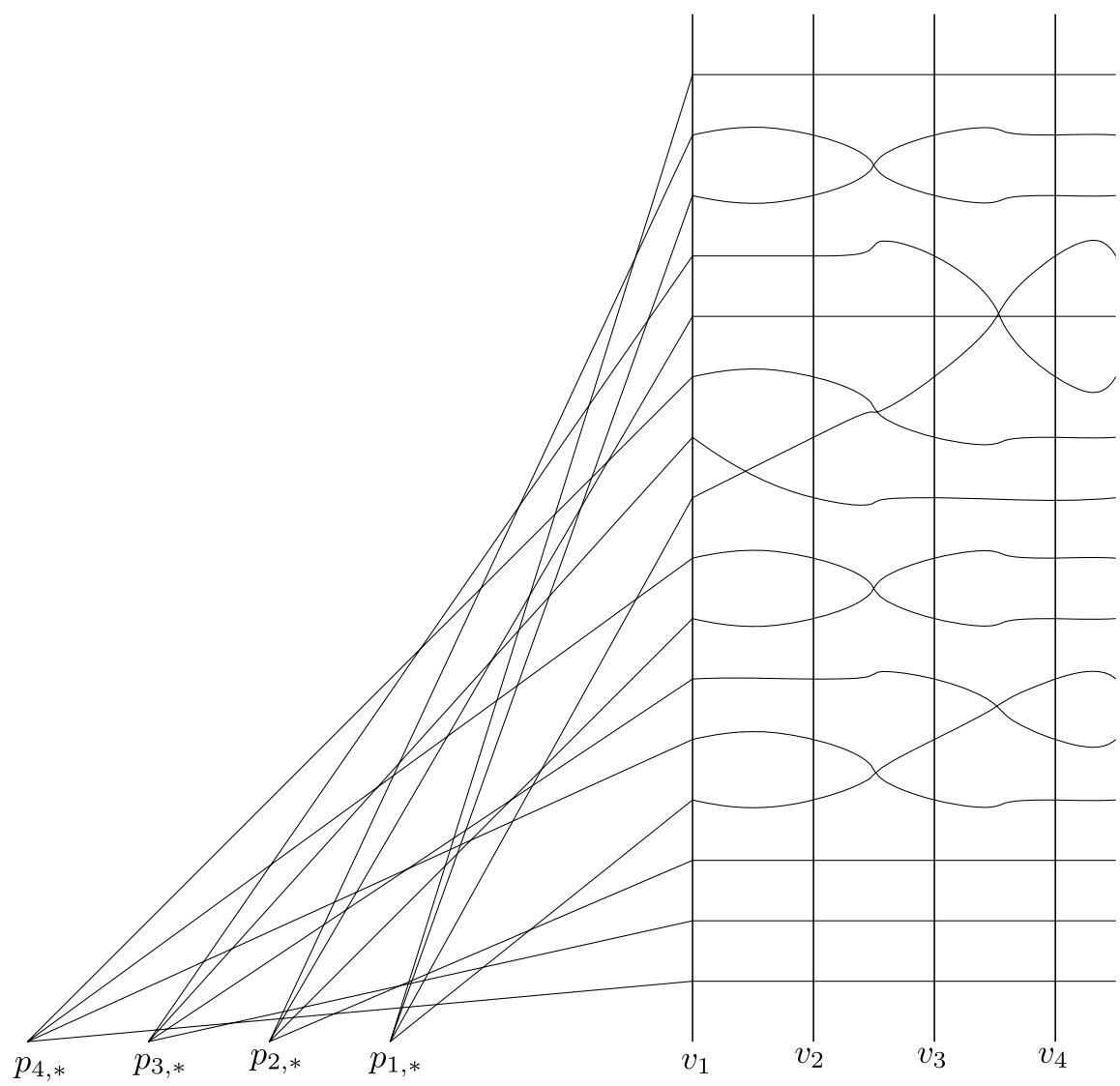

Fig. 12 Pseudoline representation of $U_{4}$

In $\hat{U}_{n}$, there were only $n$ values attained by $x$-coordinates of left endpoints of line segments (namely $-1, \ldots,-n$ ). In $\tilde{U}_{n}$, the $x$-coordinates of the left endpoints of the corresponding segments are grouped to $n$ disjoint intervals. By Lemma 5.2, this property is preserved in the $\mathfrak{p}_{2}$-representation of the poset. A similar property holds for the $x$-coordinates of the right endpoints.

Also by the lemma, the image of a pencil in the $\mathfrak{p}_{2}$-representation resembles a ray (a half-line). More precisely, the following properties hold:

- The left endpoints are confined to an interval in which no other line segments start, except the ones with identical $x$-coordinates of right endpoints.

- The right endpoints lie in the disjoint intervals mentioned in the previous paragraph; each segment has its right endpoint in exactly one interval, and the order is also preserved.

- The "shorter" line segments (in $\mathfrak{p}_{2}$ ) do not intersect any line segments that the "longer" ones do not. (Under the term "longer", we mean "farther reaching": the segments here are those whose right endpoint has greater $x$-coordinate, although their "arc length" can technically be shorter.) 
For each pencil, concentrate only the "longest" line segments. We know where these start on the $x$-axis (recall how they are confined into disjoint intervals).

We will add vertical lines to this $\mathfrak{p}_{2}$-representation. First, consider the replicas of sets $L_{i}$ for a fixed $i$ that were moved to the left. These are confined to an interval $J_{i}^{\mathrm{L}}$ in the $\mathfrak{p}_{2}$-representation. The replicas that were moved to the right are confined to the interval $J_{i}^{\mathrm{R}}$. The same holds for the original copies of $L_{i}$, call their interval $J_{i}$.

$J_{i}^{\mathrm{L}}, J_{i}$ and $J_{i}^{\mathrm{R}}$ are pairwise disjoint, and $J_{i}$ is the one in the middle. So there is a real number $r_{i}$ strictly between $J_{i}^{\mathrm{L}}$ and $J_{i}^{\mathrm{R}}$.

In the stripe $J_{i}^{\mathrm{L}} \times \mathbb{R}$ there exists a pseudoline, and Proposition 6.5 defines the order it intersects the "longest" line segments defined above. This order is $l_{i}$, the total order defined by pencil domination. There is a similar pseudoline in the stripe $J_{i}^{\mathrm{R}} \times \mathbb{R}$, on which the intersection order is the same. So the order must still be same on the vertical line with $x=r_{i}$. Keep only these vertical lines along with the "longest" line segments, and turn the "longest" line segments into lines by continuing them to infinity at both ends.

What we get is a straight line arrangement that adheres to all the defining requirements of the members of $\mathcal{F}_{n}$, contradicting the assumption that no pseudoline arrangement in $\mathcal{F}_{n}$ is stretchable.

\section{Properties of Continuous Universal Functions}

In this section, we will heavily use Lemma 5.2, and we will frequently do it implicitly, i.e., we will assume that certain segments are arranged in the specified way.

Lemma 7.1 Let $f$ be a continuous universal function and let $r, b$ be two line segments with $r^{\mathrm{L}}>b^{\mathrm{L}}$.

(i) If the right endpoint of $r$ lies on the line defined by $b$, then the right endpoint of $f(b)$ lies on the line defined by $f(r)$.

(ii) If the right endpoint of $b$ lies on the line defined by $r$, then the right endpoint of $f(r)$ lies on the line defined by $f(b)$ (specifically in the interior of $f(b)$ ).

The proof of this lemma relies on a sequence of basic statements that are claimed together in Lemma 7.2. Note that these do not require continuity.

Lemma 7.2 Let $r_{1}$ and $b_{1}$ be two line segments of a representation of a $\mathfrak{p}_{1}$ poset $P$ such that $r_{1}^{\mathrm{L}}>b_{1}^{\mathrm{L}}$. Let $b$ and $r$ be the corresponding elements of $P$. Find $a$ $\mathfrak{p}_{2}$-representation of $P$. In this, the line segments corresponding to $r_{1}$ and $b_{1}$ will be called $r_{2}$ and $b_{2}$, respectively. Let the lines defined by $r_{i}$ be called $R_{i}$ and the lines defined by $l_{i}$ be called $L_{i}$, respectively.

Then

(a) $\left(b_{1}^{\mathrm{R}}<r_{1}^{\mathrm{R}}\right) \wedge\left(r_{1} \cap b_{1} \neq \emptyset\right) \Rightarrow\left(r_{2} \cap b_{2} \neq \emptyset\right)$

(b) $\left(b_{1}^{\mathrm{R}}<r_{1}^{\mathrm{R}}\right) \wedge\left(r_{1} \cap b_{1}=\emptyset\right) \wedge\left(r_{1} \cap B_{1} \neq \emptyset\right) \Rightarrow\left(r_{2} \cap b_{2}=\emptyset\right) \wedge\left(R_{2} \cap b_{2} \neq \emptyset\right)$

(c) $\left(b_{1}^{\mathrm{R}}<r_{1}^{\mathrm{R}}\right) \wedge\left(r_{1} \cap b_{1}=\emptyset\right) \wedge\left(r_{1} \cap B_{1}=\emptyset\right) \Rightarrow\left(r_{2} \cap b_{2}=\emptyset\right) \wedge\left(R_{2} \cap b_{2}=\emptyset\right)$ 


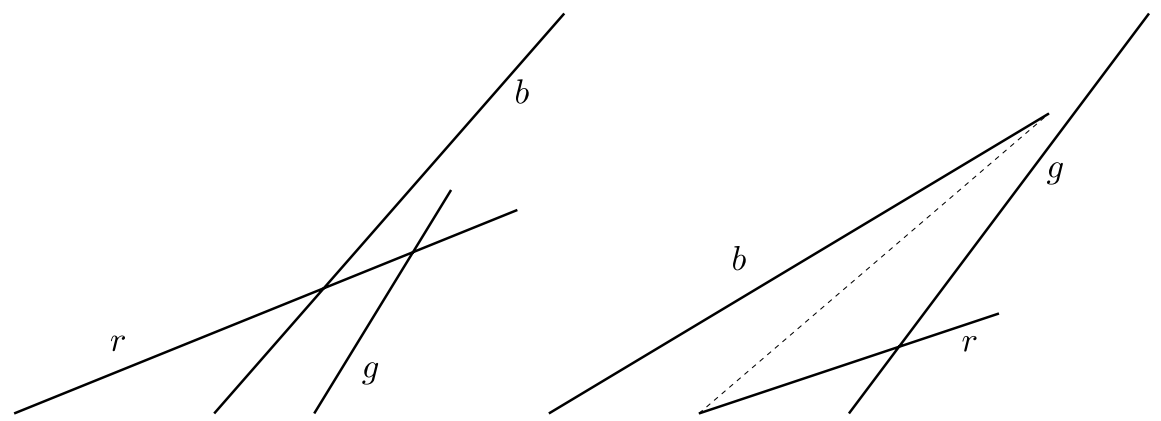

Fig. 13 Proof of Lemma 7.2 case (d)

(d) $\left(r_{1}^{\mathrm{R}}<b_{1}^{\mathrm{R}}\right) \wedge\left(r_{1} \cap b_{1} \neq \emptyset\right) \Rightarrow\left(r_{2} \cap b_{2}=\emptyset\right) \wedge\left(R_{2} \cap b_{2}=\emptyset\right)$

(e) $\left(r_{1}^{\mathrm{R}}<b_{1}^{\mathrm{R}}\right) \wedge\left(r_{1} \cap b_{1}=\emptyset\right) \wedge\left(R_{1} \cap b_{1} \neq \emptyset\right) \Rightarrow\left(r_{2} \cap b_{2}=\emptyset\right) \wedge\left(R_{2} \cap b_{2} \neq \emptyset\right)$

(f) $\left(r_{1}^{\mathrm{R}}<b_{1}^{\mathrm{R}}\right) \wedge\left(r_{1} \cap b_{1}=\emptyset\right) \wedge\left(R_{1} \cap b_{1}=\emptyset\right) \Rightarrow\left(r_{2} \cap b_{2} \neq \emptyset\right)$

Proof (a) is trivial, because if $r_{2} \cap b_{2}=\emptyset$, then $r>b$ would follow, while $r \| b$.

In (b), the part that $r_{2} \cap b_{2}=\emptyset$ is trivial for similar reasons: we must have $r>b$. To prove the other part, add a new line segment $g_{1}$ such that $b_{1}^{\mathrm{L}}<g_{1}^{\mathrm{L}}<r_{1}^{\mathrm{L}}, b_{1}^{\mathrm{R}}<$ $g_{1}^{\mathrm{R}}<r_{1}^{\mathrm{R}}$ and $g_{1} \cap b_{1} \neq \emptyset$ and $g_{1} \cap r_{1} \neq \emptyset$. Lemma 5.2 implies $b_{2}^{\mathrm{L}}<g_{2}^{\mathrm{L}}<r_{2}^{\mathrm{L}}$ and $r_{2}^{\mathrm{R}}<g_{2}^{\mathrm{R}}<b_{2}^{\mathrm{R}}$. Then $g_{2} \cap r_{2} \neq \emptyset$ because we need $g \| r$, and $g_{2} \cap b_{2} \neq \emptyset$ because we need $g \| b$. This implies $R_{2} \cap b_{2} \neq \emptyset$.

(c) will be proven at the end of the proof.

To prove (d), add a new line segment $g_{1}$ such that $g_{1}^{\mathrm{L}}<b_{1}^{\mathrm{L}}<r_{1}^{\mathrm{L}}, g_{1}^{\mathrm{R}}<r_{1}^{\mathrm{R}}<b_{1}^{\mathrm{R}}$, $g_{1} \cap b_{1}=\emptyset$ and $g_{1} \cap r_{1} \neq \emptyset$ (Fig. 13). Lemma 5.2 implies $g_{2}^{\mathrm{L}}<r_{2}^{\mathrm{L}}<b_{2}^{\mathrm{L}}$ and $r_{2}^{\mathrm{R}}<$ $b_{2}^{\mathrm{R}}<g_{2}^{\mathrm{R}}$. Since $g<b$, we have $g_{2} \cap b_{2}=\emptyset$. Since $r \| g$, we must have $r_{2} \cap g_{2} \neq \emptyset$. Therefore, the right endpoint of $r_{2}$ in $Q_{2}$ cannot stay in the triangle defined by the left endpoints of $b_{2}$ and $r_{2}$ and the right endpoint of $b_{2}$. This implies both statements.

To prove (e), add two new line segments $g_{1}$ and $w_{1}$ in the following way.

- $g_{1}^{\mathrm{L}}<b_{1}^{\mathrm{L}}<r_{1}^{\mathrm{L}}<w_{1}^{\mathrm{L}}$

- $r_{1}^{\mathrm{R}}<g_{1}^{\mathrm{R}}<w_{1}^{\mathrm{R}}<b_{1}^{\mathrm{R}}$

- $g_{1} \cap b_{1} \neq \emptyset, w_{1} \cap r_{1} \neq \varnothing$ and $w_{1} \cap b_{1} \neq \varnothing$

- $w_{1} \cap g_{1}=\varnothing$

(See Fig. 14.) By Lemma 5.2, we may assume that $r_{2}^{\mathrm{L}}<g_{2}^{\mathrm{L}}<w_{2}^{\mathrm{L}}<b_{2}^{\mathrm{L}}$ and $w_{2}^{\mathrm{R}}<$ $r_{2}^{\mathrm{R}}<b_{2}^{\mathrm{R}}<g_{2}^{\mathrm{R}}$. Since $g \| b$, we have $g_{2} \cap b_{2} \neq \emptyset$. Similarly, $r_{2} \cap w_{2} \neq \emptyset$. But $w>g$; therefore, $r_{2} \cap g_{2} \neq \emptyset$. This implies the first statement of the lemma.

To show the second statement, add $e_{1}$ such that $b_{1}^{\mathrm{L}}<e_{1}^{\mathrm{L}}<r_{1}^{\mathrm{L}}, r_{1}^{\mathrm{R}}<e_{1}^{\mathrm{R}}<b_{1}^{\mathrm{R}}$ and $e_{1} \cap r_{1} \neq \emptyset$ and $e_{1} \cap b_{1} \neq \emptyset$. By Lemma 5.2, $r_{2}^{\mathrm{L}}<e_{2}^{\mathrm{L}}<b_{2}^{\mathrm{L}}$ and $r_{2}^{\mathrm{R}}<e_{2}^{\mathrm{R}}<b_{2}^{\mathrm{R}}$. Due to the already proven part (d), $r_{2} \cap e_{2}=\emptyset$ and $e_{2} \cap b_{2}=\emptyset$. Hence $r_{2} \cap b_{2}=\emptyset$.

To show (f), add $g_{1}$ so that $b_{1}^{\mathrm{L}}<r_{1}^{\mathrm{L}}<g_{1}^{\mathrm{L}}, r_{1}^{\mathrm{R}}<b_{1}^{\mathrm{R}}<g_{1}^{\mathrm{R}}$ and $g_{1} \cap r_{1} \neq \emptyset$ and $g_{1} \cap b_{1}=\emptyset$. By Lemma 5.2, $r_{2}^{\mathrm{L}}<b_{2}^{\mathrm{L}}<g_{2}^{\mathrm{L}}$ and $g_{2}^{\mathrm{R}}<r_{2}^{\mathrm{R}}<b_{2}^{\mathrm{R}}$. Since $g \| r$, it follows that $g_{2} \cap r_{2} \neq \emptyset$. Since $g>b$, it follows that $g_{2} \cap b_{2}=\emptyset$. This implies the statement. 


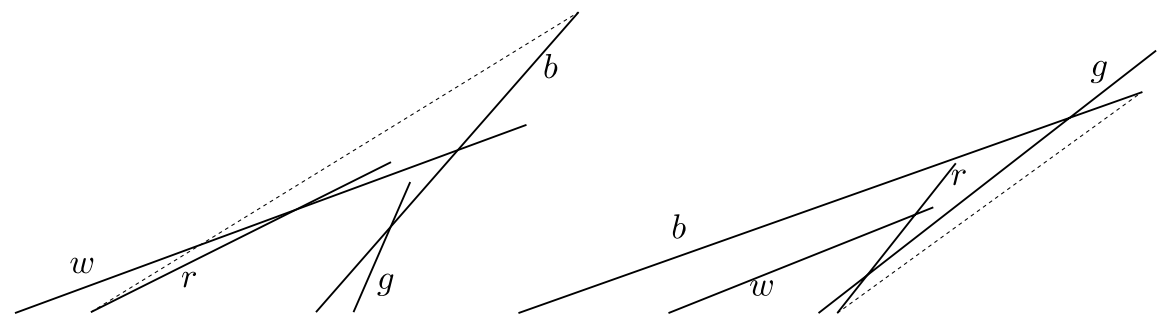

Fig. 14 Proof of Lemma 7.2 case (e)

Now let us return to (c). We get the first part trivially from $r>b$. To see the second part, add a new line segment $g_{1}$ such that $g_{1}^{\mathrm{L}}<b_{1}^{\mathrm{L}}<r_{1}^{\mathrm{L}}, b_{1}^{\mathrm{R}}<r_{1}^{\mathrm{R}}<g_{1}^{\mathrm{R}}$ and $g_{1} \cap b_{1} \neq \emptyset$ and $g_{1} \cap R_{1}=\emptyset$. Lemma 5.2 implies $b_{2}^{\mathrm{L}}<r_{2}^{\mathrm{L}}<g_{2}^{\mathrm{L}}$ and $r_{2}^{\mathrm{R}}<b_{2}^{\mathrm{R}}<g_{2}^{\mathrm{R}}$. Due to the already proven part (d), $g_{2} \cap b_{2}=\emptyset$. Because of the part (f), $g_{2} \cap r_{2} \neq \emptyset$. This implies the second part.

Now we are ready to prove Lemma 7.1.

Proof The lemma contains four statements altogether. Part (i) has two statements enclosed: one, if the right endpoint of $r$ lies in the interior of $b$, and the other, if it lies outside of $b$. Similarly, part (ii) includes two statements. All four statements have very similar proofs, so we only include the proof of the case of part (i) when the right endpoint of $r$ lies in the interior of $b$. The other statements are proven similarly.

Consider a sequence of segments $\left\{r_{i}\right\}_{i=1}^{\infty}$ such that all $r_{i}$ have their left endpoints at the left endpoint of $r$, and their right endpoints converge to the right endpoint of $r$, but the relation of $r_{i}$ and $b$ is such as for $r$ and $b$ in part (d) of Lemma 7.2. Also consider another sequence of segments $\left\{R_{i}\right\}_{i=1}^{\infty}$ such that their left endpoints are also at the left endpoint of $r$, their right end points also converge to the right endpoint of $r$; however, the relation of $R_{i}$ and $b$ is as for $r$ and $b$ in part (e) of Lemma 7.2.

Let $T$ be the triangle determined by the line segment $f(b)$ as a side and the left endpoint of $f(r)$ as the opposite vertex. The images $f\left(r_{i}\right)$ all lie outside of $T$ (left endpoints being at a vertex), and the images $f\left(R_{i}\right)$ lie inside of $T$ (left endpoints being at the same vertex). The right endpoints of these segments converge to the right endpoint of $f(r)$. So the right endpoint of $f(r)$ must lie on the boundary of $T$, which implies the statement of the lemma.

Nothing that has been done in this section depends on the position of the $y$-axis. Since Lemma 5.2 works also in the absence of the $y$-axis, and we did not use the $y$ axis in Lemma 7.2, it follows that Lemma 7.1 works also for shift insensitive universal functions. We emphasize this in the following corollary.

Corollary 7.3 Let $f$ be a continuous shift insensitive universal function, and let $r, b$ be two line segments with $r^{\mathrm{L}}>b^{\mathrm{L}}$.

(i) If the right endpoint of $r$ lies on the line defined by $b$, then the right endpoint of $f(b)$ lies on the line defined by $f(r)$. 
(ii) If the right endpoint of $b$ lies on the line defined by $r$, then the right endpoint of $f(r)$ lies on the line defined by $f(b)$ (specifically in the interior of $f(b)$ ).

\section{Continuous Shift Insensitive Universal Functions}

Lemma 8.1 Let $r$ be a line segment from $(-x, 0)$ to $(y, z)$ and $b$ be a segment from $(-x, 0)$ to $(y, w)$ with $x, y, z, w>0$ and $z>w$. Let $f$ be a continuous universal function. Then $f(r)$ and $f(b)$ have a common left endpoint and their right end points have equal $x$-coordinates, but different $y$-coordinates.

Proof Lemma 5.2 and the continuity of $f$ imply that the $x$-coordinates of the left endpoints of $f(r)$ and $f(b)$ are equal; therefore, the left endpoints are identical. Also, for similar reasons, the $x$-coordinates of the right endpoints are equal. So, unless $f(r)=f(b)$, the statement is true. Suppose $f(r)=f(b)$.

Let $g$ be a line segment starting at $(-x-1,0)$ passing through $(y, z)$ ending at $(y+1, z(x+y+2) /(x+y+1))$. Due to Lemma 7.1, the right endpoint of $f(g)$ lies at $f(r)=f(b)$. Considering Lemma 5.2, it is clear that the right endpoint of $f(g)$ must lie in the interior of $f(b)$. This makes $g$ and $b$ incomparable in the underlying poset, when the construction of $b$ and $g$ implies $g>b$.

Corollary 8.2 Let $r$ be a line segment from $(x, 0)$ to $(y, z)$ and $b$ be a segment from $(x, 0)$ to $(y, w)$ with $z>w$. Let $f$ be a continuous shift insensitive universal function. Then $f(r)$ and $f(b)$ have a common left endpoint and their right endpoints have equal $x$-coordinates, but different $y$-coordinates.

Proof The argument is the same as for the previous lemma. Instead of using Lemmas 5.2 and 7.1, we have to use their shift insensitive versions, and the argument can be repeated without change.

Now we are ready to prove the main theorem of this section.

Theorem 8.3 There is no continuous shift insensitive universal function.

Proof Consider two non-intersecting line segments in a $\mathfrak{P}_{1}$ or $\mathfrak{P}_{2}$-representation of a poset. Let $d\left(x_{0}\right)$ is the distance between the points that are defined by the intersection of the line segments with the horizontal line $y=x_{0}$. The function $d(x)$ is defined at least on some interval $[0, \varepsilon]$ for some $\varepsilon>0$. If $d(x)$ is monotonously increasing, then we say they diverge. Similarly, if $d(x)$ is decreasing, we say they converge. If they neither diverge nor converge, we say they are parallel (actually, this is not our definition).

Let $r$ be the line segment $(0,0)$ to $(1,2)$. Let $b$ be the line segment $(0,0)$ to $(1,1)$. For $\varepsilon>0$ define $b_{\varepsilon}$ to be the segment from $(0,0)$ to $(1,1-\varepsilon)$.

Because of Corollary 8.2, $f(r)$ and $f(b)$ diverge. Since $f$ is continuous, there exists $\varepsilon>0$ such that $f(r)$ and $f\left(b_{\varepsilon}\right)$ diverge. Let $r^{\prime}=f(r)$ and $b^{\prime}=f\left(b_{\varepsilon}\right)$.

Define a new line segment $l$ such that it passes through the points $(1,2)$ and $(1,1-\varepsilon)$ and its left endpoint is on the $x$-axis. This does not define $l$ completely 
because it does not describe the right endpoint, but that is, in fact, not necessary: the location of the right end point does not matter. Let $l^{\prime}=f(l)$.

Applying Lemma 7.1 to $r$ and $l$, we conclude that the right endpoint of $l^{\prime}$ lies on the line defined by $r^{\prime}$. Similarly, looking at $b_{\varepsilon}$ and $l$, we conclude that the right endpoint of $l^{\prime}$ lies on the line defined by $b^{\prime}$. Hence, the lines defined by $r^{\prime}$ and $b^{\prime}$ intersect at the right endpoint of $l^{\prime}$. This contradicts the fact that they diverge.

\section{Closing Remarks}

Naturally, we attempted to find an integer $n$ such that no pseudoline arrangement of $\mathcal{F}_{n}$ is stretchable. This actually would imply that, for all $m>n$, no pseudoline arrangement of $\mathcal{F}_{m}$ is stretchable because $\hat{U}_{n} \subseteq \hat{U}_{m}$. So we do not need to find the smallest $n$, and it is certainly more natural to try to prove the statement that, for $n$ large enough, no pseudoline arrangement of $\mathcal{F}_{n}$ is stretchable.

The problem of stretchability is difficult is general; however, we may be able to find a subset of $\hat{U}_{n}$ for large $n$ that is a known example of a non-stretchable arrangement. We unsuccessfully attempted to find three such examples: the non-Pappus arrangement, the so-called bad pentagon (see [7]) and the Desargues configuration. Unfortunately, we can only come to the following conclusion.

Theorem 9.1 The non-Pappus pseudoline arrangement is not a subset of $\hat{U}_{n}$ for any $n$.

This theorem is actually quite hard to prove, and since it is not closely related to the topic, we omit the proof.

Acknowledgement The authors wish to acknowledge the contribution of Stefan Felsner for his ideas on the subject.

\section{References}

1. Alon, N., Scheinerman, E.R.: Degrees of freedom versus dimension for containment orders. Order 5(1), 11-16 (1988)

2. Björner, A., Las Vergnas, M., Sturmfels, B., White, N., Ziegler, G.M.: Oriented Matroids, 2nd edn. Encyclopedia of Mathematics and its Applications, vol. 46. Cambridge University Press, Cambridge (1999)

3. Coxeter, H.S.M.: Introduction to Geometry, 2nd edn. Wiley, New York (1989)

4. Fishburn, P.C.: Intransitive indifference with unequal indifference intervals. J. Math. Psychol. 7, 144149 (1970)

5. Fishburn, P.C., Trotter, W.T.: Angle orders. Order 1, 333-343 (1985)

6. Füredi, Z., Hajnal, P., Rödl, V., Trotter, W.T.: Interval orders and shift graphs. In: Hajnal, A., Sós, V.T. (eds.) Sets, Graphs and Numbers. Colloq. Math. Soc. János Bolyai, vol. 60, pp. 297-313. NorthHolland, Amsterdam (1992)

7. Goodman, J.E.: Pseudoline arrangements. In: Goodman, J.E., O’Rourke, J. (eds.) Handbook of Discrete and Computational Geometry, pp. 83-109. CRC Press LLC, Boca Raton (1997). Chap. 5

8. Hiraguchi, T.: On the dimension of orders. Sci. Rep. Kanazawa Univ. 4(1), 1-20 (1955)

9. Mnëv, N.E.: The universality theorems on the classification problem of configuration varieties and convex polytopes varieties. In: Topology and Geometry-Rohlin Seminar. Lecture Notes in Math., vol. 1346, pp. 527-543. Springer, Berlin (1988) 
10. Pach, J., Törốcsik, J.: Some geometric applications of Dilworth's theorem. Discrete Comput. Geom. 12(1), 1-7 (1994)

11. Shahrokhi, F.: Personal communication

12. Shor, P.W.: Stretchability of pseudolines is NP-hard. In: Applied Geometry and Discrete Mathematics. DIMACS Ser. Discrete Math. Theoret. Comput. Sci., vol. 4, pp. 531-554. Am. Math. Soc., Providence (1991)

13. Trotter, W.T.: Inequalities in dimension theory for posets. Proc. Am. Math. Soc. 47, 311-316 (1975) 